\title{
Repurposing CCR5 inhibitors for stroke recovery
}

Stroke is a leading cause of longterm disability. Despite extensive research on the mechanisms underlying the recovery of functions after stroke, no medical therapies have been developed to promote this recovery. Now, in a new study published in Cell, S. Thomas Carmichael and colleagues have identified a translational target for stroke and traumatic brain injury, CC-chemokine receptor 5 (CCR5), a co-receptor for HIV, for which antagonists are already in clinical use.

The molecular and cellular mechanisms involved in recovery after brain injury, such as increased synaptic plasticity, are similar to those involved in learning and memory. "This similarity is seen in simple clinical observations (stroke patients seem to be 'learning' how to walk and talk again) and also in parallels in functional brain imaging and some cellular systems, such as long-term potentiation," explains Carmichael. "We started a research programme to determine if these molecular memory systems also play a part in the changes in the brain that mediate recovery after stroke, so-called 'brain plasticity.'

Inhibition of CCR5 signalling can enhance learning and plasticity processes in hippocampal and cortical circuits, so the authors analysed expression of CCR5 in mouse-derived cortical neurons and microglia during periods of acute tissue damage and recovery after stroke. Whereas CCR5 is undetectable in healthy mouse neurons, CCR5 expression in cortical neurons significantly increased 12 days after a stroke and this increase was sustained for up to 28 days.

Neuron-specific knockdown of CCR5 within the first week after stroke resulted in improved motor control assessed in gridwalk and cylinder tasks from week 1 , whereas motor deficits in control mice persisted throughout the testing period of 9 weeks.

Next, the authors assessed pharmacological inhibition of CCR5 with maraviroc, an antagonist of CCR5 used in the clinic for the treatment of AIDS. Intraperitoneal injections of maraviroc (100 mg per $\mathrm{kg}$ ) administered daily 24 hours post-stroke for 9 weeks resulted in improved motor control at 3 weeks - slightly later than the recovery observed with knockdown of CCR5 - compared with animals that received stroke and vehicle treatment.

Importantly, the authors also showed that short-term inhibition of CCR5 in the first month after stroke is enough to produce motor recovery even after the drug is no longer present, and that treatment with maraviroc is also effective in chronic stroke, starting 3 weeks after the injury. This is particularly important, as the only approved therapies for stroke have to be administered within 24 hours of stroke, which limits their use. Finally, CCR5

knockdown and maraviroc also enhanced recovery in mouse models of traumatic brain injury.

Mechanistically, CCR5 knockdown enhances signalling through activation of MAPK, DLK and transcription factor CREB. CREB mediates axonal sprouting in the presence of injury to induce a regenerative programme. Tracking changes in dendritic spine dynamics and axonal projections after stroke revealed that CCR5 knockdown and treatment with maraviroc increased the total number of spines in the pre-motor cortex, as a result of both a decline in the fraction of spines lost and a gain of new spines. The drug also increased axonal sprouting.

Finally, the authors assessed the potential of CCR5 as a target in patients. "There is a naturally occurring human loss-of-function mutation in CCR5, termed delta32," explains Carmichael. The authors analysed the cognitive assessment of 446 patients who had a stroke and identified 68 patients who harboured the mutation. Carmichael affirms that "stroke patients with this mutation recover from stroke better than matched stroke controls. This is the first description of a human gene that is associated with stroke recovery."

Given that maraviroc is a drug currently in use, clinical translation of CCR5 as a target for stroke recovery in patients might be relatively fast. "We are already in a phase II clinical trial with maraviroc for stroke recovery. We have organized the operational elements of the trial but have not yet enrolled patients," says Carmichael. Because maraviroc has poor blood-brain penetration, therapies that enhance the signalling effect of other downstream components of the pathway, such as CREB and DLK, could be potential candidates. "Isoform-specific phosphodiesterase inhibitors (such as PDE2A inhibitors and PDE10A inhibitors) are one way that this might be accomplished, as these would specifically enhance the signalling effect of these two molecules in neurons after stroke," concludes Carmichael.

M. Teresa Villanueva

ORIGINAL ARTICLE Joy, M. T. et al. CCR5 is a therapeutic target for recovery after stroke and traumatic brain injury. Cell 176, 1143-1157 (2019) 